EUROPHYSICS LETTERS

Europhys. Lett., (), pp. ()

\title{
A Note on Shell Models for MHD Turbulence
}

\author{
P. Giuliani and V. Carbone \\ Dipartimento di Fisica, Universitá della Calabria, 87036 Rende (Cs), Italy \\ (received ; accepted ) \\ PACS. 47.65+a - Magnetohydrodynamic. \\ PACS. 47.27.GS- Isotropic Turbulence.
}

\begin{abstract}
.
We investigate the time evolution of two different (GOY-like) shell models which have been recently proposed to describe the gross features of MHD turbulence. We see that, even if they are formally of the same type sharing with MHD equations quadratic couplings and conserved quantities, differences exist which are related to conserved quantities.
\end{abstract}

One of the striking features of turbulence in fluid flows is the existence of an energy cascade from large to small scales, which is usually described in the framework of the Richardson's picture 侸. Some efforts to describe the cascade through toy models or phenomenological considerations have been made in the last years [2], aimed to understand chaotic behaviour, energy spectra, intermittency, etc. Among others, Shell Models can be built up by dividing the wavevectors space $k$ into logarithmically spaced shells $\lambda^{n} \leq k / k_{0} \leq \lambda^{n+1}$ ( $\lambda$ is usually taken equal to 2), each shell being characterized by a discrete wavevector $k_{n}=\lambda^{n} k_{0}(n=0,1, \ldots, N)$, and by one single dynamical variable whose evolution is representative of the dynamics of fields belonging to the shell. The equations for these variables can be written by assuming quadratic nonlinear couplings between shells (as the original equations), and some of the coupling coefficients can be fixed by invoking the conservation of invariants in the dissipationless and force-free case. Up to now the so called GOY-model [3, 4] has been considered as the most representative to reproduce the turbulent features in fluid flows.

Shell Models for MHD turbulence have been introduced in the last years 河, and in particular the properties of GOY-like models have been investigated by Biskamp [6] and more recently by Frick and Sokoloff [7] (herein after referred to as B model and FS model respectively). Both models can be obtained as particular cases of the following set of equations $\left(v_{n}\right.$ and $b_{n}$ represent respectively the velocity and the magnetic field in dimensionless units)

$$
\begin{array}{r}
\frac{d u_{n}}{d t}=-\nu k_{n}^{2} u_{n}-\nu^{\prime} k_{n}^{-2} u_{n}+i k_{n}\left\{\left(u_{n+1} u_{n+2}-b_{n+1} b_{n+2}\right)\right. \\
\left.-\frac{\delta}{\lambda}\left(u_{n-1} u_{n+1}-b_{n-1} b_{n+1}\right)-\frac{1-\delta}{\lambda^{2}}\left(u_{n-2} u_{n-1}-b_{n-2} b_{n-1}\right)\right\}^{*}+f_{n}
\end{array}
$$

Typeset using EURO-TEX 


$$
\begin{array}{r}
\frac{d b_{n}}{d t}=-\eta k_{n}^{2} b_{n}+i k_{n}\left\{\left(1-\delta-\delta_{m}\right)\left(u_{n+1} b_{n+2}-b_{n+1} u_{n+2}\right)\right. \\
\left.+\frac{\delta_{m}}{\lambda}\left(u_{n-1} b_{n+1}-b_{n-1} u_{n+1}\right)+\frac{1-\delta_{m}}{\lambda^{2}}\left(u_{n-2} b_{n-1}-b_{n-2} u_{n-1}\right)\right\}^{*}+g_{n}
\end{array}
$$

or, in terms of the complex Elsässer variables $Z_{n}^{ \pm}(t)=v_{n}(t) \pm b_{n}(t)$, particularly useful in some solar-wind applications,

$$
\frac{d Z_{n}^{ \pm}}{d t}=-\nu^{+} k_{n}^{2} Z_{n}^{ \pm}-\nu^{-} k_{n}^{2} Z_{n}^{\mp}-\frac{\nu^{\prime}}{2} k_{n}^{-2} Z_{n}^{+}-\frac{\nu^{\prime}}{2} k_{n}^{-2} Z_{n}^{-}+i k_{n} T_{n}^{ \pm *}+f_{n}^{ \pm}
$$

where

$$
\begin{aligned}
T_{n}^{ \pm}= & \left\{\frac{\delta+\delta_{m}}{2} Z_{n+1}^{ \pm} Z_{n+2}^{\mp}+\frac{2-\delta-\delta_{m}}{2} Z_{n+1}^{\mp} Z_{n+2}^{ \pm}\right. \\
& +\frac{\delta_{m}-\delta}{2 \lambda} Z_{n+1}^{ \pm} Z_{n-1}^{\mp}-\frac{\delta+\delta_{m}}{2 \lambda} Z_{n+1}^{\mp} Z_{n-1}^{ \pm} \\
& \left.-\frac{\delta_{m}-\delta}{2 \lambda^{2}} Z_{n-1}^{ \pm} Z_{n-2}^{\mp}-\frac{2-\delta-\delta_{m}}{2 \lambda^{2}} Z_{n-1}^{\mp} Z_{n-2}^{ \pm}\right\}
\end{aligned}
$$

Here $\nu^{ \pm}=(\nu \pm \eta) / 2$, being $\nu$ the kinematic viscosity and $\eta$ the resistivity, $-\nu^{\prime} k_{n}^{-2} u_{n}$, eq. (11), is a drag term specific to $2 \mathrm{D}$ cases (see below), $f_{n}^{ \pm}=\left(f_{n} \pm g_{n}\right) / 2$ are external driving forces, $\delta$ and $\delta_{m}$ are real coupling coefficients to be determined. In the inviscid unforced limit, equations (3) conserve both pseudoenergies $E^{ \pm}(t)=(1 / 4) \sum_{n}\left|Z_{n}^{ \pm}(t)\right|^{2}$ for any value of $\delta$ and $\delta_{m}$ (the sum is extended to all the shells), which corresponds to the conservation of both the total energy $E=E^{+}+E^{-}=(1 / 2) \sum_{n}\left(\left|v_{n}(t)\right|^{2}+\left|b_{n}(t)\right|^{2}\right)$ and the cross-helicity $h_{C}=E^{+}-E^{-}=\sum_{n} \operatorname{Re}\left(v_{n} b_{n}^{*}\right)$. The values of $\delta$ and $\delta_{m}$ are fixed by imposing the conservation of another quantity which is the magnetic helicity in $3 \mathrm{D}$ or, in $2 \mathrm{D}$, the mean square magnetic potential [8]. In analogy with the fluid case [9] we can define a generalized quantity as $H_{B}^{(\alpha)}(t)=\sum_{n}(\operatorname{sign}(\delta-1))^{n}\left|b_{n}(t)\right|^{2} / k_{n}^{\alpha}$ whose conservation implies $\delta=1-\lambda^{-\alpha}$, $\delta_{m}=\lambda^{-\alpha} /\left(1+\lambda^{-\alpha}\right)$ for $\delta<1,0<\delta_{m}<1$ and $\delta=1+\lambda^{-\alpha}, \delta_{m}=-\lambda^{-\alpha} /\left(1-\lambda^{-\alpha}\right)$ for $\delta>1, \delta_{m}<0, \delta_{m}>1$. Thus two classes of MHD GOY models can be defined with respect to the values of $\delta$ : 3D-like models for $\delta<1$, where $H_{B}^{(\alpha)}$ is not positive definite; 2D-like models where $\delta>1$ and $H_{B}^{(\alpha)}$ is positive definite. This situation strongly resembles what happens in the hydrodynamic case where 2D-like $(\delta>1)$ and 3D-like $(\delta<1)$ models are conventionally distinguished with respect to a second generalized conserved quantity $H_{K}^{(\alpha)}(t)=\sum_{n}(\operatorname{sign}(\delta-1))^{n} k_{n}^{\alpha}\left|v_{n}(t)\right|^{2}$. Here the 3D and 2D cases are recovered for $\alpha=1,2$ where the ideal invariants are identified respectively with kinetic helicity and enstrophy. It should be noted that, although the hydrodynamic invariants are not conserved in the magnetic case, the equations which link $\alpha$ and $\delta$ are exactly the same for hydrodynamic and MHD models. Thus, once fixed $\alpha$ and $\delta$, it is a simple matter to find out which GOY model the MHD GOY one reduces to when $b_{n}=0$. To summarize we have that (with $\lambda=2$ ) the FS model for the $3 \mathrm{D}$ case is recovered for $\alpha=1, \delta=1 / 2, \delta_{m}=1 / 3$ and reduces to the usual 3D GOY model for $b_{n}=0$. The B 3D model is actually a 2D-like model. It is obtained for $\alpha=1, \delta=3 / 2, \delta_{m}=-1$ and reduces to a $2 \mathrm{D}$-like GOY model that conserves a quantity which has the same dimensions as kinetic helicity but is positive definite. The 2D FS and B models coincide, they are recovered for $\alpha=2, \delta=5 / 4, \delta_{m}=-1 / 3$ and reduce to the usual 2D GOY model for $b_{n}=0$.

Let us now briefly review the main results found in [7]. The authors investigate the problem of the magnetic field generation in a free-decaying turbulence, thus showing that: 1) in the $3 D$ case magnetic energy grows and reaches a value comparable with the kinetic one, in a 


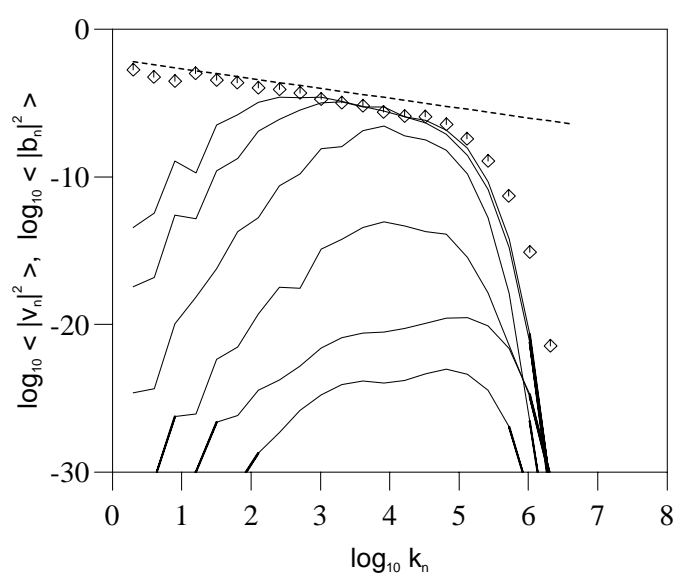

Fig. 1

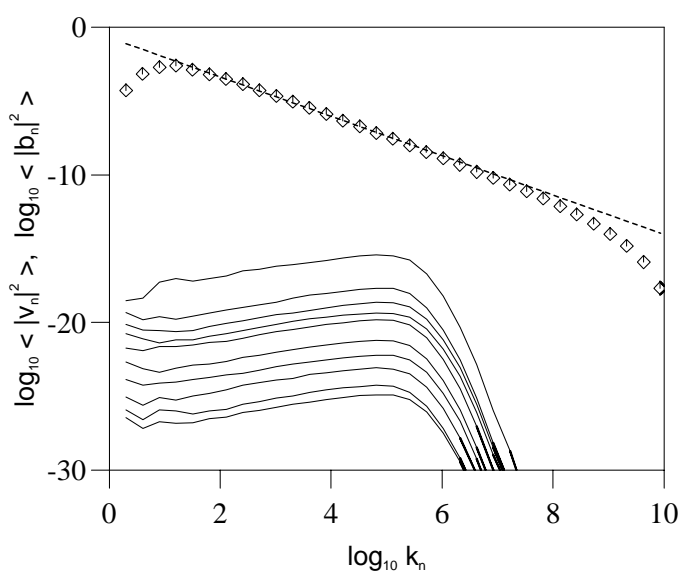

Fig. 2

Fig. 1. - FS model: $\log _{10}\left\langle\left|v_{n}\right|^{2}\right\rangle$ (diamonds) and $\log _{10}\left\langle\left|b_{n}\right|^{2}\right\rangle$ (lines) versus $\log _{10} k_{n}$. The averages of $\left|b_{n}\right|^{2}$ are made over intervals of 3 large scale turnover times. Time proceeds upwards. The kinetic spectrum is averaged over 30 large scale turnover times. The straight line has slope $-2 / 3 . \mathrm{N}=24, \nu=\eta=10^{-8}, \nu^{\prime}=0$.

Fig. 2. - B model: $\log _{10}\left\langle\left|v_{n}\right|^{2}\right\rangle$ (diamonds) and $\log _{10}\left\langle\left|b_{n}\right|^{2}\right\rangle$ (lines) versus $\log _{10} k_{n}$. Averages are made over intervals of 100 large scale turnover times. Time proceeds downwards. The kinetic spectrum is only shown for the last interval. The straight line has slope $-4 / 3$, see text for explanation. $\mathrm{N}=33, \nu=10^{-16}, \eta=0.5 \cdot 10^{-9}, \nu^{\prime}=1$.

way that the magnetic field growth is unbounded in the kinematic case; 2 ) in the $2 D$ case magnetic energy slowly decays in the nonlinear as well as in the kinematic case. These results have been interpreted as a 3D "turbulent dynamo effect" [7] and seem to be in agreement with well-known results by which dynamo effect is not possible in two dimensions [10. Morover the authors investigate the spectral properties of the model in the stationary forced case, and they find that Kolmogorov spectral properties are only established when the cross-correlation is smaller than the energy of the system. In this paper we are going to compare the results obtained from the $3 D \mathrm{~B}$ model and the $3 D \mathrm{FS}$ model as far as the "dynamo-like effect" is concerned. We started from a well developed turbulent velocity field and injected a seed of magnetic field looking at the growth of the magnetic spectra. System is forced on the shell $n=4\left(k_{0}=1\right)$, setting $f_{4}^{+}=f_{4}^{-}=(1+i) 10^{-3}$, which corresponds to only inject kinetic energy at large scales. Method of integration was a modified fourth order Runge-Kutta scheme, with a time step $10^{-4}$. In fig. 1 we report $\log _{10}\left\langle\left|b_{n}\right|^{2}\right\rangle$ and $\log _{10}\left\langle\left|v_{n}\right|^{2}\right\rangle$ versus $\log _{10} k_{n}$ for the FS model. Angular brackets \langle\rangle stand for time averages. It can be seen that the magnetic energy grows rapidly in time and forms a spectrum where the amplitude of the various modes is, at small scale, of the same order as the kinetic energy spectrum. Besides the spectral index is very close to $k^{-2 / 3}$. For a comparison we integrated the $3 \mathrm{D} \mathrm{B}$ model and it can be seen (fig. 2) that a magnetic spectrum is formed, but it slowly decays in time. Notice that, because of the smallness of $b_{n}$, its back-reaction on the velocity field is negligible, thus the kinematic part of the model evolves independently from the magnetic one. Then the scaling $\left|v_{n}\right|^{2} \sim k_{n}^{-4 / 3}$ follows, in agreement with [9] where a cascade of generalized-enstrophy is predicted for 2D-like hydrodynamic GOY models when $\alpha<2$. The question now arises whether it is correct the 


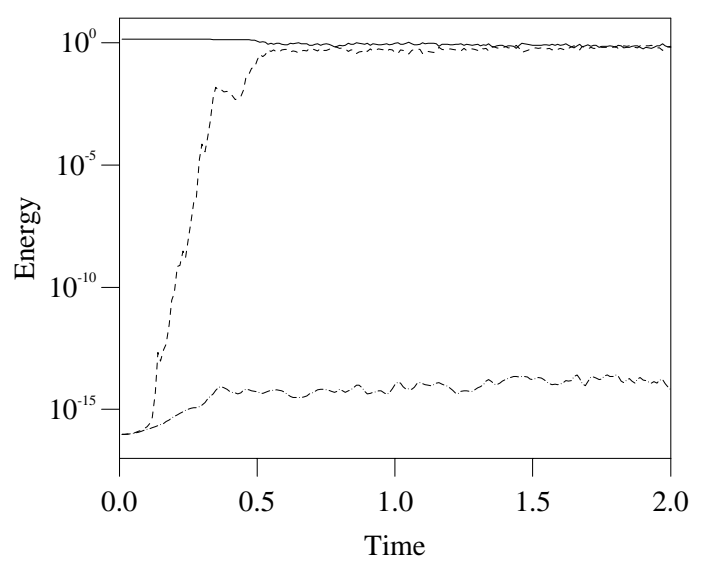

Fig. 3. - Ideal case: kinetic energy (continuous line) and magnetic energy (dashed line) versus time for the FS model; magnetic energy (dot-dashed line) versus time for the B model.

interpretation of the growth of the magnetic field in the FS model as the corresponding dynamo effect expected in the real 3D MHD. First of all it should be noted that in the kinematic case an analogy with the vorticity equation predicts the following relations between velocity and magnetic energy spectra [8]: $\left|v_{n}\right|^{2} \sim k^{-a},\left|b_{n}\right|^{2} \sim k^{2-a}$, so that if $a=2 / 3$ it follows a magnetic energy spectrum growing with $k$. The kinematic case corresponds to the first stage of growth of our simulation where this behaviour is sometimes visible, at least qualitatively. Note however that the averages are made on very small time intervals because of the rapid growth of the magnetic energy. A similar, much more pronounced behaviour is found for the B model as well. As regards the final state of evolution for the FS model, it should be reminded that equipartition of magnetic and kinetic spectra at small scales is generally attributed to the Alfvén effect, that is the propagation in opposite directions of turbulent eddies $Z_{n}^{+}, Z_{n}^{-}$along the largest scale magnetic field (see for example [8]). Note however that, as already remarked in [6] for another kind of shell models, equipartition is reached even if the Alfvénic terms are not included. The reader is referred to [6] for a thorough discussion concerning the inclusion of Alfvénic terms in shell models.

Actually we want to stress that: 1) The sign of the third ideal invariant seems to play a crucial role as far as the growth of small magnetic fields is concerned; 2) The stationary final state of evolution for the FS model does not represent a Kolmogorov-like turbulence. In fact let us consider the ideal evolution of the model $d Z_{n}^{ \pm} / d t=i k_{n} T_{n}^{ \pm *}$. We can build up the phase space $S$ of dimension $D=4 N$, by using the Elsässer variables as axis, so that a point in $S$ represents the system at a given time. A careful analysis of (3) shows that there exist some subspaces $I \subset S$ of dimension $D=2 N$ which remain invariant under the time evolution [11]. More formally, let $y(0)=\left(v_{n}, b_{n}\right)$ be a set of initial conditions such that $y(0) \in I, I$ is time invariant if the flow $T^{t}$, representing the time evolution operator in $S$, leaves $I$ invariant, that is $T^{t}[y(0)]=y(t) \in I$. The kinetic subspace $K \subset S$, defined by $y(0)=\left(v_{n}, 0\right)$ is obviously the usual fluid GOY model. Further subspaces are the Alfvénic subspaces $A^{ \pm}$defined by $y(0)=\left(v_{n}, \pm v_{n}\right)$, say $Z_{n}^{+} \neq 0$ and $Z_{n}^{-}=0$ (or vice versa). Each initial condition in these subspaces is actually a fixed point of the system. We studied the properties of stability of $K$ and $A^{ \pm}$. Following [11], let us define for each $I$ the orthogonal complement $P$, namely $S=I \oplus P$. Let us then decompose the solution as $y(t)=\left(y_{\text {int }}(t), y_{\text {ext }}(t)\right)$ where the subscripts 


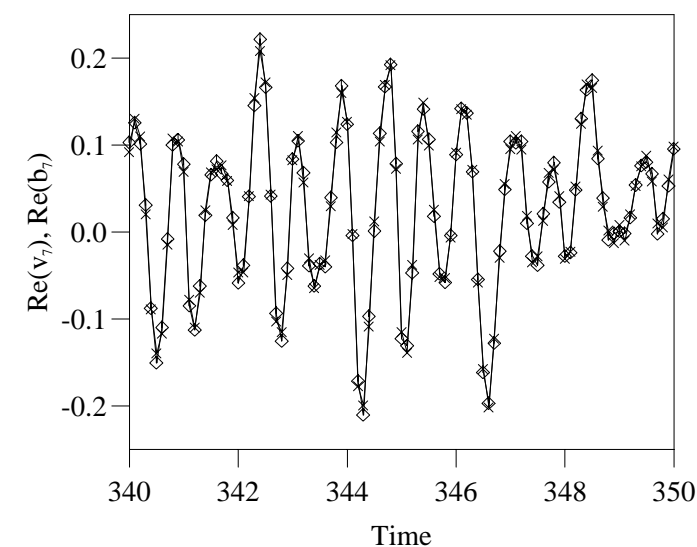

Fig. 4

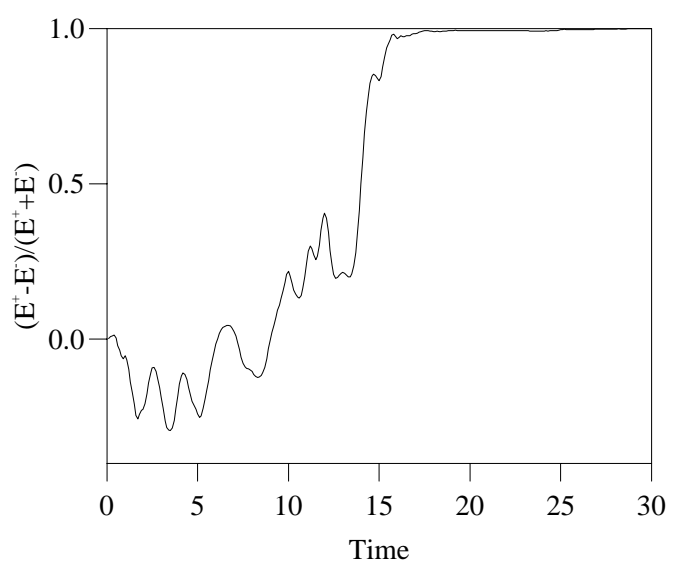

Fig. 5

Fig. 4. - FS model: $\operatorname{Re}\left(v_{7}\right)$ (diamonds) and $\operatorname{Re}\left(b_{7}\right)$ (crosses) versus time. $\mathrm{N}=19, \nu=\eta=10^{-7}$, $\nu^{\prime}=0$.

Fig. 5. - FS model: $\left(E^{+}-E^{-}\right) /\left(E^{+}+E^{-}\right)$versus time. Same parameters as in fig. 4.

refer to the $I$ and $P$ subspaces respectively. Finally we can define the energies $E_{\text {int }}=\left\|y_{\text {int }}\right\|^{2}$ and $E_{\text {ext }}=\left\|y_{\text {ext }}\right\|^{2}$. Note that the distance of a point $y=\left(y_{i n t}, y_{\text {ext }}\right)$ from the subspace $I$ is $d=\min _{\hat{y} \in I}\|y-\hat{y}\|=\left\|y_{\text {ext }}\right\|$. Then $E_{\text {ext }}$ represents the square of the distance of the solution from the invariant subspace. At time $t=0, E_{\text {ext }}=\epsilon E_{\text {int }}(\epsilon \ll 1)$ represents the energy of the perturbation. Since the total energy is constant in the ideal case, two extreme situations can arise: 1) The external energy remains of the same order of its initial value, that is the solution is trapped near $I$ which is then a stable subspace; 2) The external energy assumes values of the same order as the internal energy, that is the solution is repelled away from the subspace which is then unstable. Since the external and internal energies for the Alfvénic subspaces are nothing but the pseudoenergies $E^{+}$and $E^{-}$, which are ideal invariants, the Alfvénic subspaces are stable. As regards the kinetic subspace, $E_{\text {int }}$ and $E_{\text {ext }}$ represent respectively the kinetic and magnetic energies. Looking at the numerical solutions of the ideal model (fig. 3) we can see the difference in the stability properties between the B model and the FS model. In the first case the external energy remains approximately constant, while in the second case the system fills up immediately all the available phase space. This striking difference is entirely due to the nonlinear term, and in fact must be ascribed to the differences in sign of the third invariant. The effect of the unstable subspace, which pushes away the solutions, is what in ref. [7] is called "turbulent dynamo effect". When we introduce constant kinetic forcing and viscosity, the stable subspaces become attractors, so that the B model is attracted by its own kinetic subspace, while the FS model is attracted towards one of the Alfvénic subspaces. The dynamics of the FS model is strongly dominated by this behavior. Long runs show that the system evolves inevitably towards a "dynamical alignment" in which $v_{n}= \pm b_{n}$ as can be seen in fig. 4 where we plot $R e\left(v_{7}\right)$ and $R e\left(b_{7}\right)$ versus time. It is clearly visible how strongly velocity and magnetic field are correlated. In fig. 5 we plot, for the FS model, the time evolution of the reduced cross-helicity $\left(E^{+}-E^{-}\right) /\left(E^{+}+E^{-}\right)$, which is a global measure of the dynamical alignment. It can be seen that even from an initial value $h_{C}=0$ it grows towards unity. Due to the particular form of the nonlinear interactions in MHD (3), the nonlinear transfer 
towards the smaller scales tends to be stopped and $v_{n}$ and $b_{n}$ spectra become much steeper than that reported in fig. 1 . In this case the turbulent statistical properties are not so clear as in a Kolmogorov-like turbulence. The observed behaviour of the FS model might be avoided with a careful choice of the external driving forces. A work in this perspective is actually in progress.

We are grateful to L. Biferale, G. Boffetta and A. Celani for useful discussions, to P. Frick who gave us the preprint of his paper and to the referees whose comments have improved the final version of the paper.

\section{REFERENCES}

[1] Frisch U., Turbulence: the legacy of A. N. Kolmogorov, (Cambridge University Press) 1995.

[2] Bohr T., Jensen M.H., Paladin G. and Vulpiani A., Dynamical systems approach to turbulence, (Cambridge University Press) 1998.

[3] Gledzer E.B., Sov. Phys. Dokl., 18 (1973) 216; Yamada M. and Ohkitani K., J. Phys. Soc. Jap., 56 (1987) 4210.

[4] Jensen M.H., Paladin G. and Vulpiani A., Phys. Rev. A, 43 (1991) 798; Biferale L., Lambert A., Lima R. and Paladin G., Physica D, 80 (1995) 105.

[5] Gloaguen C., Leorat J., A. Pouquet and Grappin R., Physica D, 17 (1985) 154; Grappin R., Leorat J. and Pouquet A., J. Phys. (Paris), 47 (1986) 1127; Carbone V., Europhys. Lett., 27 (1994) 581; Frick P., Magnetohydrodynamic, 20 (1984) 262; CARBone V. and Veltri P., Geophys. Astrophys. Fluid Dyn., 52 (1990) 153.

[6] Biskamp D., Phys. Rev. E, 50 (1994) 2702; Biskamp D., Chaos, Solitons \& Fractals, 5 (1995) 1779.

[7] Frick P. and Sokoloff D., Phys. Rev. E, 57 (1998) 4155.

[8] Biskamp D., Nonlinear Magnetohydrodynamics, (Cambridge University Press) 1993.

[9] P. D. Ditlevsen and I. A. Mogensen, Phys. Rev. E, 53 (1996) 4785.

[10] Zeldovich Ya.B., Sov. Phys. JETP, 4 (1957) 460.

[11] Carbone V. and Veltri P., Astron. Astrophys., 259 (1992) 359. 